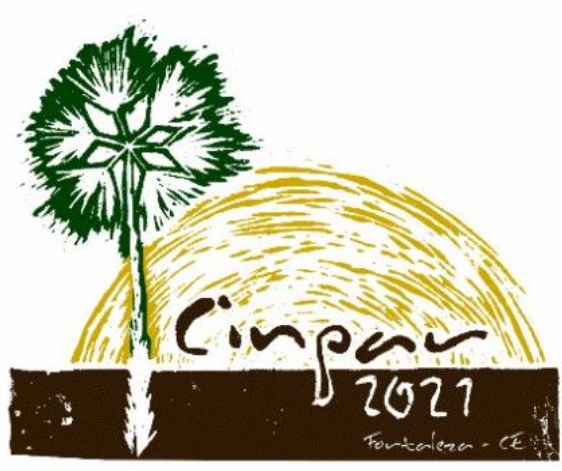

XVII Congresso Internacional sobre Patologia e

Reabilitação das Construções

XVII Congreso Internacional sobre Patología y Rehabilitación de las Construcciones

XVII International Conference on Pathology and Constructions Rehabilitation

FORTALEZA (Brasil), 3 a 5 de junho de 2021

https://doi.org/10.4322/CINPAR.2021.105

\title{
Estado da arte do estudo de fratura por fadiga em argamassas
}

\section{State of the art of fatigue fracture study in mortars}

\author{
Guilherme GOMES NOBRE ${ }^{1}$, Maykon VIEIRA SILVA², Guilherme GONZAGA PEREIRA ${ }^{3}$, Gabriel Yves da Silva \\ OLIVEIRA $^{4}$ \\ ${ }^{1}$ Universidade de Brasília, Brasil, guilhermegnobre.eng@gmail.com \\ 2 Universidade de Brasília, Brasil, eng.maykonsilva@gmail.com \\ ${ }^{3}$ Universidade de Brasília, Brasil, guilherme.gonzagaa14@gmail.com \\ ${ }^{4}$ Universidade Católica de Brasília, gabriel.yves87@gmail.com
}

Resumo: Na atualidade o estudo sobre o comportamento mecânico de estruturas de materiais cimentícios vem se tornando cada vez mais importante, pois observa que diversas manifestações patológicas são geradas por um déficit mecânico do material, sendo este, submetido a esforços no qual não foi dimensionado por falta de conhecimento do comportamento mecânico em resposta a um determinado estímulo. $\mathrm{O}$ caso das argamassas de revestimento que podem sofrer ruptura por fadiga, devido aos esforços térmicos que são submetidos ciclicamente, ou seja, a fadiga das argamassas podem ser um dos principais motivos para gerar diversas manifestações patológicas, principalmente nas de revestimento que ficam expostas a condições climáticas adversas, como variação de umidade e temperatura que podem gerar tensões de alta magnitude levando a fratura do material. Com base nisto o presente trabalho tem como objetivo descrever o estado da arte do comportamento da fratura por fadiga em argamassas, onde se fez um estudo detalhado sobre diversos trabalhos que estudaram esse mecanismo em compostos cimentícios, principalmente os que foram submetidos a diversos ciclos de carga e descarga relacionando os fatores que influenciaram nos resultados obtidos pelos autores, evidenciando que o material poderá alcançar a ruptura mesmo em tensões abaixo da tensão resistente do material.

Palavras-chave: Fratura, Fadiga, Patologia, Argamassa.

Abstract: Nowadays the study on the mechanical behavior of structures of cementitious materials has become increasingly important, as it observes that several pathological manifestations are generated by a mechanical deficit of the material, which is submitted to efforts in which it was not dimensioned due to lack of knowledge of mechanical behavior in response to a given stimulus. The case of coating mortars that can suffer from fatigue rupture, due to the thermal stresses that are subjected cyclically, that is, the fatigue of mortars can be one of the main reasons for generating several pathological manifestations, mainly in the coating that are exposed to conditions adverse climatic conditions, such as variations in humidity and temperature that can generate high magnitude stresses leading to fracture of the material. Based on this, the present work aims to describe the state of the art of fatigue fracture behavior in mortars, where a detailed study was made on several works that studied this mechanism in cementitious compounds, mainly those that were subjected to several load cycles and discharge relating the factors that influenced the results obtained by the authors, showing that the material can reach rupture even at stresses below the material's resistant tension.

Keywords: Fracture, Fatigue, Pathology, Mortar. 


\section{Introdução}

O uso de revestimento argamassado ganhou notoriedade devido sua importância em proteger os elementos estruturais da ação direta dos agentes agressivos, auxiliando as vedações quanto ao cumprimento de suas principais funções como: estanqueidade, isolamento térmico, acústico, estabilidade, durabilidade e principalmente a valorização do imóvel, contribuindo pela estética da fachada.

Para Alves (2002), os sistemas de revestimentos argamassados são utilizados com grande frequência nas fachadas de todos país. Embora observe o largo emprego destes materiais, existem poucas definições normativas. Disso resulta um significativo empirismo nas definições, especificações e produções dos sistemas de revestimento de argamassa. Devidos a estas caracterísitcas, há uma grande presença de manifestações patológicas dentro do espectro da falta de conhecimento científico do tema.

De acordo com Baía e Sabattini (2008), para que os revestimentos de argamassa possam cumprir adequadamente suas funções, eles precisam apresentar um conjunto de propriedades específicas, relativas à argamassa tanto no estado fresco quanto no estado edurecido. Por isso, Suellen (2009) constatou diante de ponderações que o revestimento argamassado é composto por materiais que apresentam características físico-mecânicas diferentes, devido às falhas apresentadas as camadas do revestimento. Assim, o revestimento argamassado tem uma grande importância em seu conjunto por se tratar da camada de suporte de todo o revestimento, levando em consideração que os revestimentos estão aderidos integralmente e qualquer deformação nas suas camadas ocasionam tensões em todas camadas presentes no sistema.

Uchôa (2007) diz que é importante notar que o revestimento cerâmico é geralmente empregado nas fachadas das edificações com o auxílio de diferentes materiais, que possuem, consequentemente, diferentes características físico-mecânicas. Tais diferenças, são causadas por um conjunto de ações, entre elas, a insolação, podendo produzir deformações e tensões nas fachadas de origem termomecânicas e podem gerar sérios problemas patológicos, em virtude da grande variação de deslocamentos (dilatação térmica). Fiorito (1994), enfatiza que, apesar de o sistema de revestimento cerâmico estar sujeito a tensões de diversas naturezas atuando simultaneamente, destaca-se aquelas de origens térmicas, são enfaticamente, reportadas como principal resposável pelo desplacamento de peças cerâmicas, seja nas principais idades ou após longo período.

Diante do grande desenvolvimento brasileiro na produção de peças cerâmicas, aumentam a quantidade e o volume dos aparecimentos das manifestações patológicas neste sistema e Possan e Demoliner (2013) afirmam que a degradação precoce das edificações e consequente redução de desempenho são problemas frequentes. Esta degradação ocorre pelo envelhecimento precoce dos revestimentos argamassados, que é desencadeado pela qualidade inferior dos materiais de construção utilizados, por problemas de projeto, execução e falta de manutenção.

Sendo assim, Uchôa (2007) diz que o processo de fadiga é um fenômeno físico caracterizado pela deterioração progressiva e irreversível do material, verificado após a aplicação de certo número de ciclos de carregamentos. Já Kisher e Dutta (2020), para modelar o comportamento de fadiga de um material, faz-se necessário analisar a quantificação dos danos causados por cargas cíclicas. Vários parâmetros, como taxa de deformação, energia dissipada e deformação plástica acumulada.

De acordo com Naderi e Khonsari (2012, 2013 ) propuseram a entropia como uma medida de degradação por fadiga e através da realização de uma série de experimentos, foi demonstrado que a entropia gerada na falha é uma constante e pode ser considerada uma propriedade do material. Para Callister e Rethwisch (2016), a fadiga sob tais circunstâncias, é possível de ocorrer uma falha sob um nível de tensão considerávelmente inferior ao limite de resistência à tração de um certo material, levando em consideração que Chagas (2009) diz que, a argamassa de emboço é considerada um material frágil e, portanto, tem sua ruptura à fadiga no regime elástico.

Ainda de acordo com Callister e Rethwisch (2016), as tensões aplicadas que geram a fadiga podem ser aplicadas de natureza axial (tração-compressão), flexão (dobramento) ou de torção, onde essas tensões são caracterizadas através de gráficos simétricos senoidais em relação ao tempo. Ainda, existe outro gráfico que pode ser gerado, denominado de ciclo de tensões repetidas, onde relaciona valores máximos e mínimos de 
forma assimétrica em relação ao nível de tensão igual a zero, ou seja, o nível de tensão pode variar aleatóriamente em amplitude e frequência.

Por isso, o objetivo deste trabalho será de relacionar os problemas de fadiga das argamassas de revestimento, que são as falhas presentes nas estruturas que são submetidas a tensões dinâmicas e variáveis, e para alcançar estes objetivos, procedeu-se a análise de trabalhos publicados, onde estudaram o efeito da fadiga em compostos cimentícios, principalmente em argamassas que foram submetidas a diversos ciclos de carga e descarga.

\section{Metodologia}

A abordagem dessa revisão no que diz respeito ao estado da arte do comportamento da fratura por fadiga em argamassas, com intuito de análise de diversos trabalhos que relataram o comportamento destas argamassas submetidas aos ciclos de carga e descarga.

Inicialmente, definiu-se para a pesquisa em bancos de dados palavras-chave como: Fratura, Fadiga, Patologia, Argamassa ou até mesmo artigos e livros relacionados à fadiga de materiais metálicos e argamassados para compreender com clareza o efeito de fadiga de um modo geral. Durante a seleção, foi dado preferencia aos trabalhos atuais publicados nos mais conceituados congressos e principais pesquisadores que estão trabalhando sobre o assunto ao longo de suas carreiras científicas.

\section{Desenvolvimento}

De acordo com Callister (2010) fratura pode ser definida como a separação de um elemento em duas ou mais partes, ocasionada, geralmente, pelo processo de fissuração resultante de fatores externos e internos, como: aplicação de tensões no elemento (seja de tração, compressão, cisalhamento, torção ou até mesmo combinação entre as mesmas); temperatura na qual o elemento está submetido; módulo de deformação do elemento entre outros. Dentre os possíveis modos de fraturas em elementos, destaca-se a fratura por fadiga, decorrente da aplicação de tensões cíclicas, seja nos primeiros ciclos de carga, após muitos ou até mesmo não ocorrer durante a vida útil da estrutura. (ABNT, 2014 - NBR 6118, Anexo C)

Uchôa (2007) complementa afirmando que a fadiga ocorre através de uma propagação de uma trinca ou fissura, conforme a aplicação do carregamento, onde, fissuras com comportamento considerado dúctil, possuem evolução relativamente lenta quando comparadas com fissuras que se apresentam com comportamento de ruptura frágil, que se surgem de forma demasiada e rapidamente evoluem. (CALLISTER, 2010)

O comportamento do material entorno da fissura ou trinca é abordado na área de estudo relacionada à mecânica da fratura, ainda que haja uma complexidade com relação à solicitação em uma trinca ou fissura, estas em um elemento sólido podem apresentar-se, simplifacadamente, a partir de três modos de carregamento: modo de abertura, de cisalhamento no plano e de cisalhamento fora do plano (rasgamento) (UCHOA, 2007).

No estudo de comportamento do material submetido à fadiga é extremamente importante a determinação da vida à fadiga do mesmo, representado pela letra $\mathrm{N}$, definida como o número de ciclos neecessários para a ruptura do material em um nível de tensão específico. (CHAGAS, 2009)

Em revestimentos argamassados a fadiga pode acarretar manifestações patológicas em sistemas de revestimentos cerâmicos, como o desplacamento de peças, provocadas pelas tensões térmicas verificadas nas interfaces. (DA SILVA, et. al, 1998)

A resistência à fadiga está relacionada a diversos fatores, variando conforme o material, como: concentrações de tensões; rugosidade da superfície; frequência de carregamento; histórico de carregamento/ temperatura e condições ambientais. (UCHOA, 2015)

Influência da frequência do carregamento no processo de ruptura por fadiga 
Tepfers (1982) através de um ensaio dinâmico que o mesmo desenvolveu, verificou que a tensão aplicada, as suas direções e a direção na qual ela é variável influencia diretamente no comportamento à fadiga da argamassa, entretanto o efeito era muito pequeno, não sendo possível descrevê-lo em um modelo.

Outro fator que influencia nos resultados de fadiga apresentados em argamassas é a frequência, Zhang (1996) verificou que quanto maior for a frequência de carregamento maior será a resistência à fadiga do concreto, que corresponde a quase $80 \%$ da resistência estática do material. Esse comportamento também pode ser adotado em argamassas de revesimento, admitindo-se, que ambos possuem baixa ductilidade e comportamento explicitamente frágil. (CHAGAS, 2009)

Entretanto Murdock (1965) descreve que frequências de carregamentos com valores entre 1 e $15 \mathrm{~Hz}$ não possuem influência na resistência à fadiga do concreto quando a tensão máxima usada no ensaio é menor que $75 \%$ da resistência estática do concreto. Entretando, relacionar-se a frequência, de acordo com banco de dados analisados, é pouco abordado.

\section{Influência da variação da temperatura na resistência à fadiga}

A ação da temperatura e sua devida variação é ligada diretamente à dialatação térmica do material e seu devido coeficiente, em relação à exposição contante aos intempéries e incidência solar.

Dentre os trabalhos que se destacam na pesquisa do efeito térmico em argamassas de revestimento e revestimento cerâmico está o de Uchôa (2007). O autor realizou, através de um arcabouço de dados metereológicos, a influência e a variação local da temperatura da cidade de Brasília nos esforços internos gerados que resultaram em fratura por fadiga, onde o mesmo, através de dados de máxima e mínimas históricas da cidade, correlacionou à variação da temperatura à vida por fadiga de argamassas. 0 programa experimental baseou-se em diferentes transientes térmicos e tonalidades do revestimento cerâmico que influenciaria diretamente na absorção térmica dessas e da propagação que este efeito ocasionaria em todo o sistema de revestimento (substrato, chapiso, emboço e revestimento cerâmico). O autor relata que para o caso analisado a maior amplitude térmica destaca-se na faixa das $16 \mathrm{~h}$, onde o transiente térmico é mais acentuado em todos os casos estudados pelo mesmo. $O$ efeito da variação térmica é descrita pelo autor como o causador de dois diferentes esforços no sistema de revestimento cerâmico. O primeiro é quando o aumento gradativo da temperatura, quando comparada com a temeperatura ambiente, ocasiona no sistema esforços internos de tração, devida à expansão térmica, gerando esforços cisalhantes na interface dos elementos que compõem o sistema. Em contrapartida a queda gradual da temperatura, resultando em uma temperatura inferior à temperatura ambiente, ocasaiona esforços de compressão entre os elementos, resultando no desplacamento do revestimento cerâmico, devido à retração térmica do conjunto.

Este segundo fator apresentado por Uchôa é descrito por Fiorito (1994), onde este, por sua vez, cita que em determinados instantes, a depender do nível, a variação térmica ocasiona tensão de compressão no revestimento. Podendo ocorrer o rompimento, a partir de uma sequência de oscilações térmicas.

Da Silva et al (1998) simulou através de um modelo numérico, utilizando elementos finitos, uma fachada de um ediíficio, buscando obter as tensões que podem ocorrer no sistema de revestimento cerâmico, gerados por variações extremas de temperaturas. Os resultados obtidos pelo autor fora o esperado e semelhante aos resultados de Uchôa (2007), a exposição de sistemas de revestimento cerâmicos à ciclos de variações térmicas, extremas ou moderadas, geram esforços de tração e compressão internamente, gerando assim patologias no sistema, como desplacamento de peças cerâmicas, ocasionadas por esforços de compressão gerados pelo efeito de retração gerados na argamassa colante e no revestimento argamassado (emboço).

Apresenta-se como fator carente de pesquisas detalhadas e relevância, que não constam no banco de dados utilizado, a influência do módulo de elasticidade da argamassa, devendo esta, de acordo com Thomaz (1989) ser preponderantemente superior ao módulo de deformação do substrato.

Chagas (2009) descreve o comportamento de dois tipos de argamassa, industrializada e mista, expostas à variações cíclicas, gerando assim o fenômeno de fadiga, comparando-as através da curva S-N. Nos resultados do programa experimental proposto, para o nível de tensão mínimo às quais os CP's foram submetidos (70\%) ambas as argamassas obtiveram valores distintos para o número de ciclos no qual ocorreu a fratura das mesmas. A argamassa indistrializada conseguiu suportar, a partir de uma tensão de 7,13 MPa, 1350084 ciclos de exposição, enquanto a mista, submetida a uma tensão de 5,98 MPa, necessitou de 1191729 ciclos ate a fratura. Nota-se uma diferença de aproximadamenteo $19 \%$ na tensão aplicada e de $13 \%$ a mais de ciclos 
necessários para o rompimento da industrializada. Portanto, a partir dos resultados, destaca-se a maior capacidade resistente a fadiga da argamassa industrializada, onde a mesma foi submetida a uma tensão maior e necessitou de mais ciclos para que ocorresse a ruptura. Os valores apresentados foram os valores máximos de ciclos que cada argamassa suportou.

\section{Influência da concentração de tensões na resistência à fadiga}

O fator de concentração de tensões nos ensaios experimentais de fadiga são parte primordial no que diz respeito a resistência da argamassa ou concreto à fadiga, além disso este fator é influenciado pela heterogeneidade do material. $O$ fator é designado através da letra $K_{t}$ que é a relação tensão máxima sobre tensão média, diferentemente do fator de intensidade de tensões $K$, usado para definir a magnitude do campo de tensões (CHAGAS, 2009). O mesmo depende de relações geométricas de alguns parâmetros do corpo de prova (UCHOA, 2007)

Uchoa (2007) em seus ensaios experimentais correlaciona o fator $\mathrm{K}_{\mathrm{t}}$ e as dimensões do corpo de prova (CP) moldado pelo autor. O CP moldado possui largura determinada e pré-estabelecida (D) da região retangular, com um entalhe tambem com parte retangular de largura (d) e com zona de transição semicircular de raio (r). De acordo com o autor era esperado que a ruptura ocorresse no entalhe, onde ocorreria, teoricamente, a maior concentração de tensão. $\mathrm{O}$ autor demonstrou que o fator $\mathrm{K}_{\mathrm{t}}$ cresce exponencialmente conforme diminui a relação $\mathrm{r} / \mathrm{d}$.

\section{Outros fatores não listados que influenciam na resistência à fadiga especificamente em revestimentos cerâmicos}

SARAIVA (1998) em seu trabalho descreve em seus resultados experimentais fatores que influenciam diretamente a resistência à fadiga em revestimentos cerâmicos, são eles: espessura da camada de emboço; influência do coeficiente de absorção térmica e a influência do tipo de rejunte.

O autor, através de uma análise estatística realizada, descreve como a variação dos parâmetros listados anteriormente refletem nos resultados experimentais. Para o primeiro fator variou a camada de emboço para 3 casos, sendo para cada, respectivamente, 2,cm; $1,0 \mathrm{~cm}$ e 5,0 cm de espessura.

De acordo com Saraiva (1996), apresenta uma definição simplificada ao comportamento dos revestimentos cerâmicos ao receber esforços, onde variou-se o tipo de argamassa colante, espessura da camada de emboço, coeficiente de absorção térmica da cerâmica e tipo de rejunte. Através disso, após análises dos resultados de tensões normais recebidas ao longo do eixo $\mathrm{X}, \mathrm{Y}$ e cisalhamento entre as camadas do sistema. A discussão final relatou sobre os principais fatores de influenciaram as tensões obtidas a partir da variação numérica realizada por Saraiva (1996), com o objetivo de compreender as causas principais geradas por diferentes resultados de tensões a partir de uma única variável, considerando a variação de espessura da camada de emboço, coeficientes de absorção térmica da cerâmica, tipo de rejunte e influência do tipo de argamassa colante utilizada.

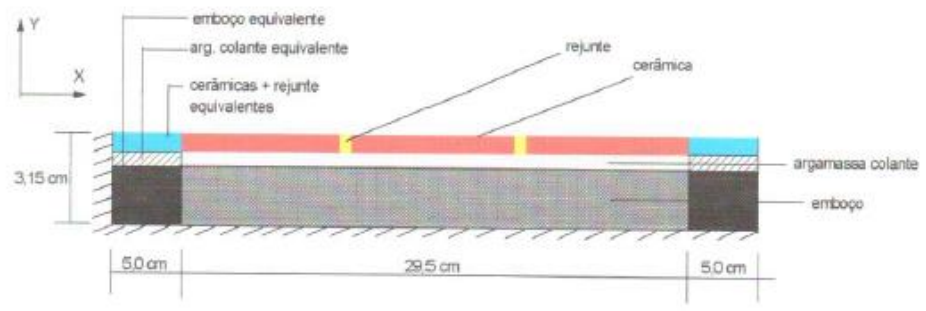

Figura 1: Modelo ideal para obtenção de resultados

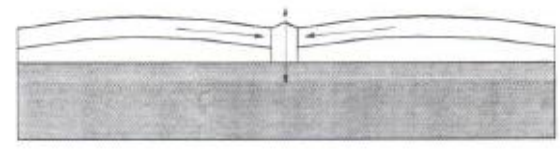

Figura 2: Comportamento na aregião nas juntas, mostrando a tendência à traçao no rejunte 


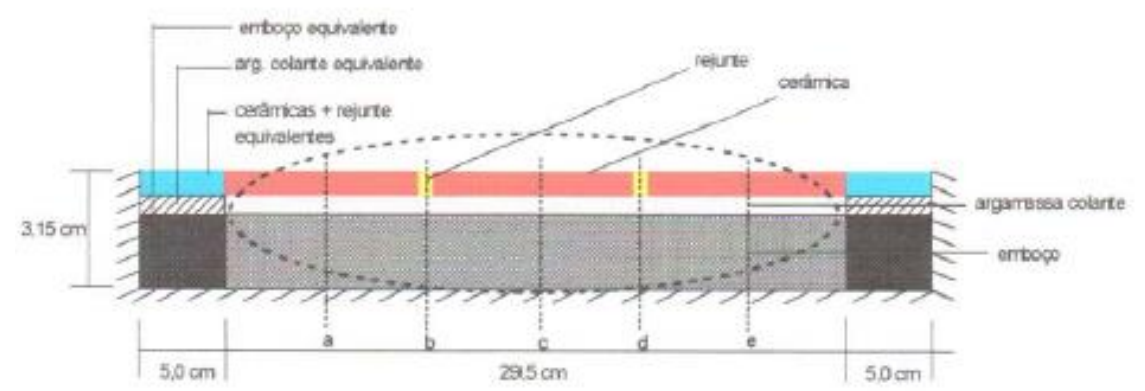

Figura 3: Modelo exemplificado: a região em destaque é o objeto em destaque

Portanto, em relação ao eixo X observou-se que em todas as camadas (camada de peças cerâmicas e rejunte, argamassa colante e emboço) dos sistema compostos por argamassa colantes tipo A e B, há um aumento de $60 \%$ nas tensões de compressão normais ao longo do eixo X. Diante disso, observou que apenas na camada composta por argamassa colante houve uma diferença significativa dos valores das tensões entre sistemas compostos, sendo maiores para o sistema composto por argamassa colante tipo A que, como já foi justificado, possui um maior módulo de elasticidade.

Para o eixo $Y$, na interface das camadas de argamassa colante/emboço são maiores para o sistema composto por argamassa colante tipo A. Na região abaixo das juntas ( pontos b e d) as tensões são positivas na interface argamassa colante/peças cerâmicas e rejunte, e negativa entre argamassa colante/emboço. Por isso, assim como as tensões do eixo $X$, um aumento entorno de $60 \%$ nos valores das tensões ao longo do eixo $Y$, devido ao aumento do carregamento em questão (temperatura).

Para as tensões de cisalhamento, devido ao maior módulo de elasticidade, assim como as tensões normais ao longo do eixo $\mathrm{X}$ e $\mathrm{Y}$, houve um aumento de aproximadamente de $60 \%$ nos valores de tensões de cisalhamento, onde também esta relacionado ao composto da argamassa colante $A$, devido ao maior módulo de elasticidade.

Deixa-se evidente a necessidade do ensaio de determinação do módulo de resistência à flexão, descrito em detalhes no anexo C da NBR 13818, devido ao esforço de flexão a que as peças cerâmicas estão submetidas, devido ao aumento das tensões na região das juntas e diminuição no centro das peças cerâmicas.

Para as tensões de cisalhamento, houve aumento nos valores entre a argamassa colante/peças cerâmicas e rejunte e entre argamassa colante/emboço, sendo este aumento mais significativo para o sistema composto por argamassa colante tipo B.

Para Toyama et al (2017), estabeleceu um modelo computacional de uma fratura de contato em argamassa de concreto submetida a ensaios de indentação monotônica e cíclica com indentador esférico, com o intuito de imitar as fraturas de contato locais. Diante disso, foi possível comprovar danos de microescala nas fraturas de contato e pelo teste de indentação monotônica, comprovou que a fissura se propaga com o aumento do número de ciclos, considerando a mesma carga aplicada. Assim $\mathrm{O}$ método dos elementos finitos (FEM) foi empregado para elucidar as distribuições de tensões de início e propagação de trincas durante ambos os carregamentos de indentação. Potanto, verificou-se que o crescimento de trincas por fadiga sob indentação cíclica pode ser simulado e o modelo desenvolvido pode ser útil para examinar a propagação de trincas em outros materiais frágeis sob cargas de contato monotônicas e cíclicas.

Ainda, de acordo com Bauer et al (2012), o estudo desenvolvido, podem-se enumerar as seguintes principais conclusões: $O$ fenômeno da fadiga atua sobre as argamassas de revestimento e pode reduzir fortemente as resistências à tração das argamassas; As patologias físico-mecâncias observadas nas fachadas dos edifícios, submetidas à ciclagem térmica, dentre outras, podem ter origem na redução das resistências mecânicas pelo efeito de fadiga; Frequentemente avalia-se a compatibilização das argamassas e revestimentos cerâmicos somente ponderando as propriedades estáticas, particularmente o módulo de elasticidade. É também fundamental analisar também as reduções nos valores de resistências pelo feito da ciclagem (carga-descarga) que caracterizam o comportamento de fadiga. 
Silva (2019), diz que como as fissuras são anomalias que apresentam várias possibilidades para sua formação, sendo, assim, inevitáveis, principalmente, em camadas argamassadas, recomenda-se, com base na avaliação de fadiga das camadas do revestimento, o emprego de placas cerâmicas com baixo nível de absortância em estruturas de revestimentos cerâmico. As estruturas portadoras de cerâmicas brancas não apresentam risco de ruptura, mesmo com a presença de fissuras em suas camadas. Logo, as fissuras representam um fator de contribuição para o crescimento das tensões no interior do revestimento, e não um ponto determinante para o processo de ruptura da camada, tendo em vista que as estruturas com baixo nível de absortância não apresentaram ruptura em suas camadas argamassadas. E ainda, foi possível observar que apesar de certos casos apresentarem ruptura com elevados números de ciclos, ficou evidente que o carregamento térmico foi capaz de gerar níveis de tensões superiores a resistência à fadiga de compressão das camadas.

Alencar (2020), constatou que as argamassas colantes de uma forma geral apresentam maior vida útil à fadiga com a redução da relação entre tensão aplicada e sua tensão de ruptura estática; As argamassas com menores módulos de elasticidade apresentam maior vida à fadiga e as com maiores módulos os piores resultados e que as argamassas apresentam rupturas bruscas durante os ensaios de fadiga (cíclico), sem desenvolvimento a propagação de fissuras visíveis, mostrando comportamento frágil diante deste tipo de solicitações. $O$ fato ainda de não ter evidenciado com um bom entendimento um patamar de resistência à fadiga, não significa que não o possuam. Porém, materiais cimentícios como argamassas e concreto não parecem possuir este tipo de patamar, devendo ser atribuído um limite de projeto.

\section{Considerações finais}

O presente artigo é uma síntese do estudo da fadiga em revestimentos argamassados, sendo que sua influência atinge até os revestimentos cerâmicos, onde o objetivo primordial era relacionar as principais falhas ocasionados principalmente pela ação da fadiga do material em análise. O material disponivel na literatura se mostrou escasso, pois os principais estudos com relação a fadiga se dá em materiais metálicos, dificultando uma revisão bibliográfica, mas a partir do material disponível sobre o assunto podemos concluir que esse tema é de fundamental importância. A fadiga é o responsável por sérios danos em fachadas, causando assim prejuízos de desempenho e de custos para a edificação, sabendo que a fachada é o sistema que protege a edificação das intempéries e dos agentes degradantes. $O$ estudo da fadiga é essencial para a prevenção de anomalias que causaram danos maiores se forem protelados, mostrando-se assim o necessário contínuo no avanço dos estudos de fadiga em materiais cimentícios e em revestimentos, para a prevenção e um diagnóstico mais assertivo com relação as anomalias. Para pesquisas futuras, é importante salientar a relevância do estudo da influência da frequência de aplicação de cargas no ensaio de fadiga de argamassas e suas variações de resultados, considerando o equívoco de avaliar o comportamento de fadiga exposto à variação de temperatura com um ciclo curto (24h). Deve-se considerar um tempo maior para chegar nos resultados esperados, fazendo o uso também de análise higrotérmica para verificação das patologias e profundidade das fissuras geradas. Avançoes importantes vem sendo gerado, relacionando a fadiga dos materiais com base nas características de fadida na adesão de betume em argamassas. Sendo assim, para o estudo proposto ainda deve um avanço significativo, principalmete para o avanço de resultados com equipamentos e maquinários para um resultado preciso.

\section{Referências Bibliográficas}

Alencar, D. R. L. (2019), Análise experimental da fadiga mecânica em argamassas colantes. Tese de mestrado. Universidade de Brasíilia, Brasil.

Alves, N.J. (2002). Avaliação dos aditivos incorporadores de ar em argamassas de revestimento. Tese de Mestrado. Faculdade ed Tecnologia, Universidade de Brasília, Brasil.

Associação Brasileira de Normas Técnicas. NBR 15575 - Desempenho de Edificações Habitacionais. Rio de Janeiro 2013.

Baía, L.L.M., Sabbatini, F.H., (2002). Projeto e execução de revestimento argamassa. O Nome da Rosa, ISBN 85-86872-14-8, São Paulo, Brasil.

Título abreviado do artigo, usando no máximo 100 caracteres incluindo os espaços em branco 
BAUER, E., BEZERRA, L. M., UCHÔA, J. C. B., CHAGAS, S. V. M. Estudo do comportamento à fadiga das argamassas e sua relação com as patologias e danos nas fachadas. 4. - Congreso de patología y rehabilitación de edificios. 12-14 de abril de 2012, Santiago de Compostela, 2012, ISBN 978-84-96712-49-2, pág. 63

Callister, W.D., Rethwisch, D.G. (2012). Ciência e Engenharia de Materiais: uma introdução. LTC, 8ª Ed, Rio de Janeiro, Brasil.

Chagas, S.V.M., (2009). Estudo de Proposta de um Modelo de Resistência à Fadiga de Argamassas de Revestimento em Estado Plano e Tridimensional de Tensões. Faculdade de Tecnologia. Universidade de Brasília, Brasil.

DAS CHAGAS, S. V. M. (2009). Estudo e Proposta de um Modelo de Resistência à Fadiga de Argamassas de Revestimento em Estado Plano e Tridimensional de Tensões. Dissertação de Mestrado em Estruturas e Construção Civil, Publicação E.DM - 015 A/09, Departamento de Engenharia Civil e Ambienal, Universidade de Brasília, Brasília, DF, 172p.

Dutta, S., Kishen, J.M.C. (2019). Mesoscale analysis od Fatique Damage through Aggregate-Mortar Bond Cracks in Cementitious Composites. American Society of Civil Engineers - ASCE. DOI: 10.1061/(ASCE)EM.1943-7889.0001706.

Fiorito, A.J.S.I., (1994). Manual de Argamassa de Revestimento - Estudo e Procedimento de Execução. Pini, São Paulo, Brasil.

Long Li., Z.W., Song, L., Guo, S., Dai, Q. (2019) High-Frequencu Fatique Performance of Cracked Mortar After Epoxy Grouting Reinforcement. American Society of Civil Engineers - ASCE. DOI: 10.1061/(ASCE)GM.19435622.0001416.

Naderi, M., Khonsari, M.M., (2013). On the role of damage energy in the fatigue degradation characterization of a composite laminate. Composites Part B: Engineering. DOI: 10.1016/j.compositesb.2012.07.028.

Nascimento, M.L.M. (2016). Aplicação da simulação higrotérmica na investigação da degradação de fachadas de edifício. Tese de Mestrado. Faculdade de Tecnologia, Universidade de Brasília, Brasil.

Mo, L., Huurman, M., Wu, S., Molenaar, A. A. A. (2007), Ravelling investigation of porous asphalt concrete based on fatigue characteristics of bitumen-stone adhesion and mortar. Materials and Design, Wuhan, China.

Liu, G., Van der Ven, M., Wu, Shaopeng., Yu, JianYing, Y., Molenaar, A. (2011). Influence of organo-

montmorillonites on fatigue properties of bitumen and mortar. International Journal of Fatigue. Wuhan,

China.

Pacheco, C.P., Vieira, G.L., (2017). Análise quantitativa da degradação das fachadas com revestimento cerâmico. SciELO. Programa de Pós-Graduação em Engenharia Civil, Centro Tecnológico. Vitória, Brasil.

Possan, E., Demoliner, C.A., (2013). Desempenho, Durabilidade e Vida Útil das Edificações: Abordagem Geral. Revista Técno-Científica do CREA-PR. Curitiba, Brasil.

Pu, Q., Yang, S., Shi, Z., Houng, Y., Zhou, Y. (2020). atique Performance of an innovative Steel-Concrete joint in Long-Span railway hybrid box girder cable-stayed bridges. American Society of Civil Engineers - ASCE. DOI: 10.1061/(ASCE)BE.1943-5592.0001680.

SARAIVA, A. G., 1998. Contribuição ao Estudo de Tensões de Natureza Térmica em Sistemas de Revestimento Cerâmico de Fachada. Dissertação de Mestrado, Publicação E.DM 004A/98, Departamento de Engenharia Civil, Universidade de Brasília, Brasília, DF, 164p.

Saraiva, A.G. (1998). Contriuição ao estudo de de tensões de natureza térmica em sistemas de revestimento cerâmico de fachadas. Tese de Mestrado Faculdade de Tecnologia, Universidade de Brasília. Brasil.

Soares, E. M., Antunes, E. G. P. (2017), Avaliação da resistência de aderência à tração de argamassas colantes industrializadas com adição de inibidor de hidratação. Tese de conclusão de curso de Engenharia Civil. Universidade Extremo Sul Catarinense, Brasil.

Silva, L. V. P. (2019), Modelagem Numérica de Estrutura de Revestimento fissurado: Avaliação da degradação e fadiga. Tese de mestrado. Universidade de Brasília, Brasil. 
Toyama, H., Kishida, H., Yonezu, A. (2017), Characterization of fatigue crack growth of concrete mortar undercyclic indentation loading. Engineering Failure Analysis, Tokyo, Japan.

UCHÔA, J. C. B. (2007). Procedimento Numérico e Experimental para a Avaliação da Resistência à Fadiga de Sistemas de Revestimento. Dissertação de Mestrado em Estruturas e Construção Civil, Publicação E.DM002a07, Departamento de Engenharia Civil e Ambiental, Universidade de Brasília, Brasília, DF 159p.

UCHÔA, J. C. B. (2015). Análise Numérica e Experimental da Fadiga Termomecânica em Argamassas Colantes no Sistema de Revestimento Cerâmico. Tese de Doutorado em Estruturas e Construção Civil, Publicação E. TD-005 A/15, Departamento de Engenharia Civil e Ambiental, Universidade de Brasília, Brasília, DF, 214p. 